\title{
Application of Leo Bernard's "Inherent Stimulus" Theory in Poster Design
}

\author{
Yao Zhang ${ }^{1}$, Bing Liu 2, a, * \\ ${ }^{1}$ Academy of Fine Arts, Northeast Normal University, Changchun130024, China \\ a ice8515@163.com \\ ${ }^{*}$ Corresponding author
}

Keywords: "Inherent Stimulus" theory, Poster design, Application.

\begin{abstract}
The theory of natural stimulus of Leo Bernard was studied, and combined with business cases, the principles of its application in poster design are analyzed in detail. The results show: The theory of "intrinsic stimulation" has an important application in the poster design, and it shows the following three aspects, the cause of the production of the enterprise, the selling point of the product and the inducement of the consumer's purchase. In the poster design, we should fully explore the inherent drama of the product, that is, combining the surrounding environment, with the purpose of product manufacturing and consumer demand, constantly renewing its own ideas, slogans and even tone, seeking the suitable poster design elements, and make it fit with sales market, consumers' needs and desires. The theory of inherent stimulus gives the poster new connotation, making it a special form of expression with unique artistic charm.
\end{abstract}

\section{Introduction}

With the rapid development of the political economy and the dramatic changes in the social environment, especially the wide application of electronic information technology, the social value of posters is rising day by day; direct dissemination of information and the richness of its contents show people a wonderful visual feast. The creative strategy of "inherent stimulus", which was put forward by Leo Burnett in $60 \mathrm{~s}$, was unique, with this unique creative strategy, he succeeded in saving the "Marlboro cigarettes", this theory is also the representative of the Chicago school, and also the spirit of today's advertising creative people, it is also widely used in poster design [1].

\section{Theoretical analysis}

\subsection{An overview of the "intrinsic stimulation method"}

"Inherent stimulus" is also called "innate drama", that is, the most important task of advertising creativity is to excavate and make use of the inherent stimuli, "drama" can be summed up as: "make the enterprise produce it, make people buy its essence"[2], "inherent stimulus" is a timely coincidence of product production purpose and promotional content with consumer demand, it includes two main contents: first, the causality between the three purposes of product production, publicity content and consumer demand, it is the intrinsic factor of the "drama" of the product, the chain of cause and effect coexists with time and personnel, so the "drama" of the product will never disappear, but it is difficult to find, the two is the commodity sale opportunity which includes time, place, political trend, economic environment, social fashion and so on, it is the external condition of "drama". When the internal factors match the external conditions, the "drama" of the product is revealed, and the sales of products continue to expand and occupy favorable markets.

The purpose of manufacturing, the content of propaganda and the demand of the consumer are a kind of relationship, cause and effect in the process of manufacturing and selling products, and also a process of continuous development and change, the purpose of product manufacturing is because of 
its abstract and broad nature, but also due to the slow change of product production, and the development of the object and content and consumer demand at any time, very active, the "drama" of the product is shown in the change of the two, therefore, they are the core elements of the "dramatic" product, the so-called "innate drama" is actually the core element as a clue to seek and discover the various factors that relate to the needs of the consumer in the characteristics and utility of the product $[3,4]$.

\subsection{The overview of the poster design}

Poster, also known as "poster", is a way and medium to transmit information in a plane and visual form, the poster design is a visual communication language composed of elements such as graphics, symbols, words, colors and arrangement, the final purpose of the posters is to disseminate information, it is the link between the communicators and the recipients, and it is rich, it also has great exploration space and rules of expression.

With the development of new technology and new media, the traditional poster design will not only disappear, but become more important, the form of poster design will be more diversified, the poster design will stand on the people's visual stage, the important meaning of the poster design is to make the poster a kind of thought and culture. A special art of design, with aesthetic value and free spirit, occupies a prominent position in the wave of design, and has become a medium of communication with unique artistic charm [5].

\subsection{The art of relevance between "inherent stimulus" and poster design}

The famous Leo Benner "intrinsic stimulation" theory plays a very important role in the poster design, which not only enriches the visual expressiveness of the poster design itself, but also excavates a more diverse creative form, the theory of "intrinsic stimulation" points out that it is necessary to excavate the innate drama of things, and to give a new image to things on this basis, and to express them in a new relationship, poster design is to recombine known and believable things and create a new meaningful relationship between seemingly unrelated things, the demands of the two are consistent, creating a new relationship on the basis of the inherent characteristics, and displaying the characteristics of things with some fresh and original ideas.

In the poster creation, the image is a special form of the design works reflecting the real life, it is the creative embodiment of the poster design in the works, it is also one of the most important elements in the graphic design, including the poster creation, how to vividly and effectively depict the visual objects of the creative objects becomes the primary task, this is associated with the inherent relationship image inherent in the innate digging of things in Leo Burnett "intrinsic stimulation", creating a simple and powerful image, but each has its own "inner drama", but each product has its own "internal drama", and the intrinsic stimulation of the product itself can start with the three aspects of the reasons for the production of the product, the selling point of the product and the incentives of the consumers.

\section{Application of "inherent stimulus" theory in poster design}

\subsection{Analysis of KIBON ice cream poster}

Renata EL Dib designed a group of poster for the Brazil ice cream company KIBON in the form of curling the corner of the poster to simulate ice cream cone shape, showing the reel form of chocolate, original flavor, and strawberry ice cream in the poster, as shown in figure 1. the font is symmetrical on the top of the poster, showing the ice-cream brand clearly, in the background color of the poster, chocolate, white and pink are used to show different flavors of ice cream, which makes people feel the sweetness of ice cream, using the back of the poster to display ice cream, the production process is simple, giving people a unique and innovative visual impact, the posters used curly expression to expand the two-dimensional space to the three-dimensional space, which greatly enriched the visual expression of the poster. 
The ice-cream and crisp canisters are integrated into the poster, making people feel cool and tasty. The poster excavates the consumer's purchase incentives, first, using the shape of the product to display the poster with three-dimensional method, it gives the audience a sense of shock in vision and sense of touch; second, the pictures of each poster are all ice cream, which can not only effectively arouse the attention of the consumers, seize the sight of the consumers, but also raise the desire of the consumers, thus achieving a good visual publicity effect; third, using the material of the product to design the back of the poster as a sweet tube, simply and directly express the raw material of the product, which can arouse the customer's good sense of the product and cause the potential purchase incentive, this group of poster shows "the inherent stimulus inherent in the product itself is based on the desire and interest of the consumer".
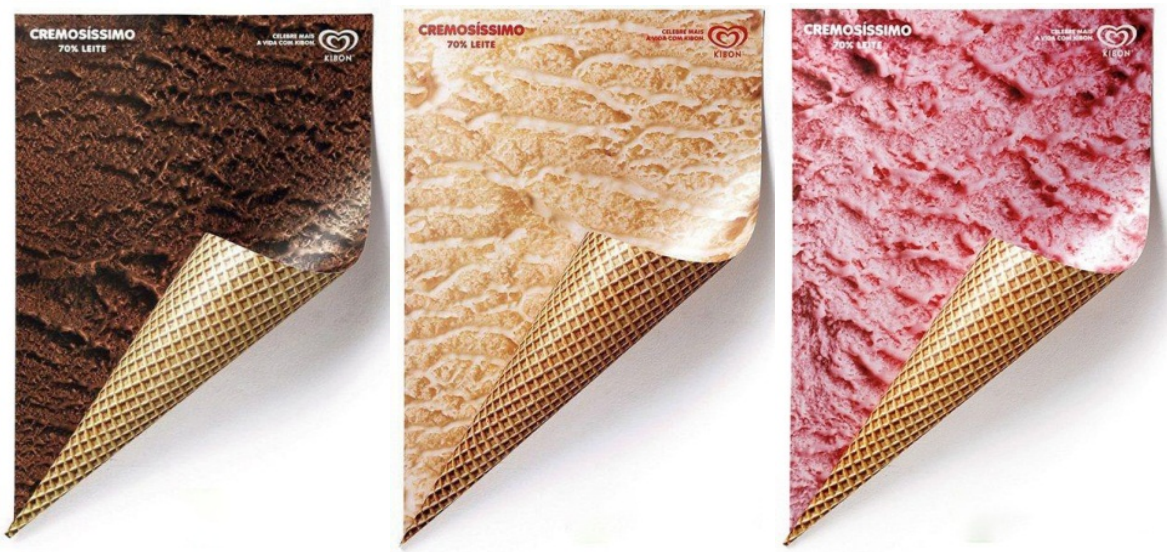

Fig. 1. The poster of KIBON ice cream

\subsection{Analysis of HEINZ poster for tomato sauce}

The first poster of HEINZ ketchup uses a stable composition method, the main part is in the visual center of the picture, and the bottom is advertising language and logo, the distribution of theme and advertising language is very reasonable, the slogan on the poster clearly expressed the theme of creativity, highlighting the uniqueness of the product, which is impossible for other similar products, the red color of the poster is echoed with the color of tomatoes. Red represents passion, vitality, health and strong visual impact, the original flavor of HEINZ tomato sauce is vividly expressed by using the creative expression technique of reengineering imagination, using the bottle bottom and tomato to make up the complete image of tomato.

In this poster design, dig out the selling point of the product itself, and display the inherent irritation of the product itself in front of the consumers. First, the use of the shape of the product to display, the bottom of the bottle and tomato, bottle and tomato slices combined, both full of interest and emphasize the theme of publicity; the second is to use the color of the product to excavate, the color relationship is very coordinated and unified, the main color red keeps the overall beauty of the picture; third, from the raw material of the product, use tomato and product to carry on creative association, make the viewer reappear the corresponding visual image in the brain according to the actual image of the tomato.

\subsection{Analysis of the poster of Tefal Juicer}

In this group of Tefal Juicer poster design, the central composition method was used to highlight the main part, the black background of the poster matches the color of pure color, it gives a bright and gorgeous visual feeling, and the contrast is more obvious, which shows the theme of the lively, fresh, the creative point of this poster is to use intuitive creative graphics to show that the juicer can meet your fresh and fast requirements, simple and accurate transmission of information, it is easy to seize the eyes of people in an instant, as shown in figure 2.

In this group of Tefal Juicer poster design, fully demonstrated the reasons for the production of products. First, from the function of the product, the body of pineapple, carrot and radish in the poster is turned into fruit juice, indicating that the juicer can make $100 \%$ pure juice also prove that other 
enterprises can't do it; second, from the point of view of market demand, it can squeeze juice from fruits and vegetables, and achieve the fresh, healthy and natural pursuit of consumers; third, from the enterprise culture, it shows that the customer is the service tenet of God, so it is not difficult to see that the enterprise seeks to improve and keep pace with the times, and tries to create a satisfactory product for consumers.
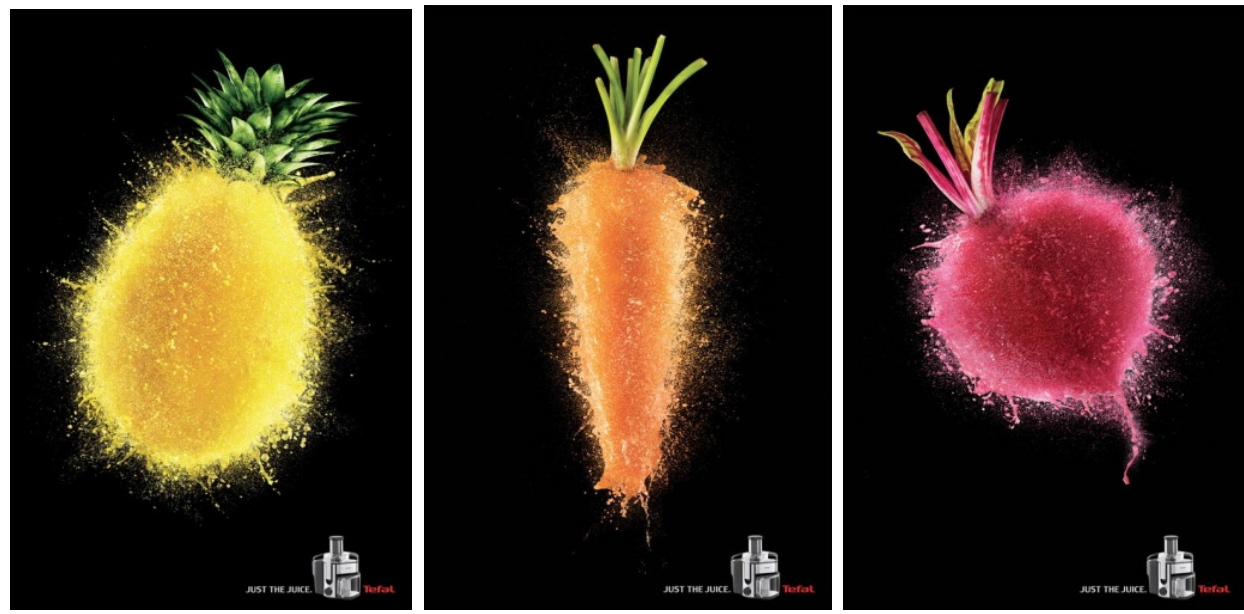

Fig. 2. The posters of Tefal Juicer

\section{Conclusion}

Leo Burnett's "inherent stimulus" theory points out not only to explore the internal drama of the product, but also to combine the environment around it, to inject new content and to show it in a new form, the core elements of the "dramatic" product play an important communication role in the "propaganda content, the poster design", which is based on the purpose of the product manufacturing, and its pursuit is to meet the needs of the consumer. We should constantly update their ideas, slogans and even tone in order to find the appropriate elements of the poster design so that they can be successfully expressed in the sales market, the needs and desires of the consumers. With the development of modern society, poster design plays a very important role in real life, the designer can fully learn the theory of Leo's "intrinsic stimulation" and apply it to the poster design, which adds a lot of interest to the poster design, which enables us to reexamine the diversity of the posters' design forms, as well as promote the sales of the products and improve the social concern of the products.

\section{Acknowledgement}

Youth fund of Northeast Normal University in philosophy and social science projects - (2017QT008).

\section{References}

[1] Bruce Bendinger, Advertising Copybook Training Manual, China Media University Press, pp. 42-45, 2008. (in Chinese)

[2] Luo Wenkun, Advertising Psychology, Tianma Press, pp. 209-210, 1982. (in Chinese)

[3] John Hewitt, Design the poster in England,1890-1914, Early Popular Visual Culture, vol.5, pp. 57-70, 2007.

[4] Gerry A. Turner, Commercial Poster Design, Design, vol. 51, pp.8-23, 2013.

[5] Jang, Mee-Kyung, A Comparative Study on Relationship between Advertising Sociology and Illustration-Focused on the Image of Modern Women in Commercial Poster in Korea and China, Archives of Design Research, vol.18, pp.27-36, 2005. 